\title{
The Diameter of Almost Eulerian Digraphs
}

\author{
Peter Dankelmann* \\ School of Mathematical Sciences \\ University of KwaZulu-Natal \\ Durban 4000, South Africa \\ dankelma@ukzn.ac.za
}

\author{
L. Volkmann \\ Lehrstuhl II für Mathematik \\ RWTH Aachen University \\ 52056 Aachen, Germany \\ volkm@math2.rwth-aachen.de
}

Submitted: Oct 9, 2008; Accepted: Oct 20, 2010; Published: Nov 19, 2010

Mathematics Subject Classification: 05C12, 05C20

\begin{abstract}
Soares [J. Graph Theory 1992] showed that the well known upper bound $\frac{3}{\delta+1} n+$ $O(1)$ on the diameter of undirected graphs of order $n$ and minimum degree $\delta$ also holds for digraphs, provided they are eulerian. In this paper we investigate if similar bounds can be given for digraphs that are, in some sense, close to being eulerian. In particular we show that a directed graph of order $n$ and minimum degree $\delta$ whose arc set can be partitioned into $s$ trails, where $s \leqslant \delta-2$, has diameter at most $3\left(\delta+1-\frac{s}{3}\right)^{-1} n+O(1)$. If $s$ also divides $\delta-2$, then we show the diameter to be at most $3\left(\delta+1-\frac{(\delta-2) s}{3(\delta-2)+s}\right)^{-1} n+O(1)$. The latter bound is sharp, apart from an additive constant. As a corollary we obtain the sharp upper bound $3\left(\delta+1-\frac{\delta-2}{3 \delta-5}\right)^{-1} n+O(1)$ on the diameter of digraphs that have an eulerian trail.
\end{abstract}

Keywords: digraph, eulerian, semi-eulerian, diameter.

\section{Introduction}

While for undirected graphs bounds on the diameter have been well researched, much less is known about the diameter of directed graphs. Most bounds on the diameter of undirected graphs do not have a straightforward analogue for directed graphs. A case in point, and the starting point of our investigation, is the following well-known bound on the diameter on an undirected graph in terms of order and minimum degree.

Theorem 1 Let $G$ be a connected graph of order $n$ and minimum degree $\delta \geqslant 3$. Then

$$
\operatorname{diam}(G) \leqslant \frac{3}{\delta+1} n+O(1)
$$

Apart from the additive constant, this bound is best possible.

${ }^{*}$ Financial support by the South African National Research Foundation is gratefully acknowledged 
As shown by Soares [3], the above bound does not hold for digraphs. He constructed graphs of order $n$, minimum degree $\delta$ (defined as the minimum over all in-degrees and all out-degrees) and diameter $n-2 \delta+1$. His construction shows that for fixed $\delta$ there is no constant $a_{\delta}<1$ such that all strongly connected digraphs of minimum degree $\delta$ and sufficiently large order have diameter at most $a_{\delta} n+O(1)$. On the other hand, Soares showed that the bound in Theorem 1 does hold for eulerian digraphs, i.e., for digraphs in which every vertex has the same in- and out-degree.

This raises the natural question if relaxations of the eulerian property still allow us to give meaningful bounds on the diameter in terms of order and minimum degree. Two ways of relaxing the eulerian property seem obvious candidates: (i) consider digraphs in which the difference between in-degree and out-degree of a vertex is bounded by a constant, and (ii) consider digraphs whose arc set is the union of a bounded number of trails. For both relaxations we investigate if there exist constants $a_{\delta}<1$ such that for all such digraphs $D$ of order $n$ and minimum degree at least $\delta$, we have

$$
\operatorname{diam}(D) \leqslant a_{\delta} n+b
$$

for some constant $b$. It will turn out that for the first relaxation there is no such bound with $a_{\delta}<1$, while for the second relaxation such a bound exists.

Let $s$ be a nonnegative integer. We define a strong digraph $D=(V, A)$ to be $s$-eulerian if $\sum_{v \in V}\left|\mathrm{~d}^{+}(v)-\mathrm{d}^{-}(v)\right| \leqslant 2 s$. So $D$ is 0-eulerian if and only if $D$ is eulerian, and for $s \geqslant 1$ $D$ is $s$-eulerian if and only if the arc set of $D$ can be decomposed into $s$ trails. Note that 1-eulerian digraphs are often called semi-eulerian. For fixed $s$ and $\delta>0$ we define $c_{\delta, s}$ to be the smallest constant such that for all $s$-eulerian digraphs of order $n$ and minimum degree $\delta$

$$
\operatorname{diam}(D) \leqslant c_{\delta, s} n+b,
$$

for some constant $b$. For eulerian digraphs, i.e., for $s=0$, it follows from Soares' result that $c_{\delta, 0}=\frac{3}{\delta+1}$. For general values of $s$, the determination of $c_{\delta}$ seems to be non-trivial. In this paper we show that

$$
c_{\delta, s} \leqslant 3\left(\delta+1-\frac{s}{3}\right)^{-1} \text { if } s \leqslant \delta-2 .
$$

If $s$ divides $\delta-2$, then we give the exact value:

$$
c_{\delta, s}=3\left(\delta+1-\frac{(\delta-2) s}{3(\delta-2)+s}\right)^{-1} \quad \text { if } s \text { divides } \delta-2 .
$$

We note that the diameter of eulerian oriented graphs has been investigated in [2] and $[1]$.

\section{Results}

Before considering $s$-eulerian digraphs, we first show that there exist digraphs of order $n$, minimum degree $\delta$, and diameter $n-\delta^{2}+\delta+1$, in which the in-degree and the out-degree of every vertex differ by not more than 1 . This shows 
Proposition 1 For each $\delta \geqslant 1$ there exist infinitely many strong digraphs $D$ with the property

$$
\left|\mathrm{d}^{+}(v)-\mathrm{d}^{-}(v)\right| \leqslant 1 \text { for all vertices } v
$$

and

$$
\operatorname{diam}(D) \geqslant n-\delta^{2}-\delta+1
$$

where $n$ is the order of $D$.

Proof. Let $D$ be the directed graph of order $n$ obtained from the disjoint union of two copies $H_{1}$ and $H_{2}$ of the complete digraph $K_{\left(\begin{array}{c}\delta \\ 2\end{array}\right)}$ and a directed path $P=v_{1}, v_{2}, \ldots, v_{n-\delta^{2}+\delta}$, with arcs added from each $v_{i}$ to its $\delta$ predecessors $v_{i-1}, v_{i-2}, \ldots, v_{i-\delta}$ if $i \geqslant \delta+1$, and to $v_{i-1}, v_{i-2}, \ldots, v_{1}$ if $i \leqslant \delta$, as follows. Add arcs between the first $\delta-1$ vertices of $P$ and $H_{1}$ such that each of the $\delta-1$ vertices of $P$ has in-degree and out-degree equal to $\delta$, and each vertex of $H_{1}$ is incident to at most one arc joining it to $P$ and at most one arc joining it from $P$. Similarly add arcs between the last $\delta-1$ vertices of $P$ and $H_{2}$. It is easy to verify that the resulting digraph is strong and has diameter at least $n-\delta^{2}-\delta+1$.

This example shows that even if the in-degree and out-degree of every vertex differ by not more than 1 , then no $a_{\delta}<1$ exists such that the diameter is bounded from above by $a_{\delta} n+O(1)$.

We now turn our attention to $s$-eulerian digraphs.

Theorem 2 Let $D$ be a strong s-eulerian digraph of order $n$ and minimum degree $\delta$. (a) If $s \leqslant \delta-2$ then

$$
\operatorname{diam}(D) \leqslant 3\left(\delta+1-\frac{s}{3}\right)^{-1} n+O(1) .
$$

(b) If $s$ divides $\delta-2$ then

$$
\operatorname{diam}(D) \leqslant 3\left(\delta+1-\frac{(\delta-2) s}{3(\delta-2)+s}\right)^{-1} n+O(1) .
$$

Proof. We fix a vertex $v$ of out-eccentricity $d=\operatorname{diam}(D)$. If $i$ an integer, then we let $V_{i}$ be the $i$ th distance layer, i.e., the set of vertices at distance exactly $i$ from $v$. By $V_{\geqslant i}$ and $V_{\leqslant i}$ we mean the set of vertices at distance at least and at most $i$, respectively, from $v$. We also let $n_{i}=\left|V_{i}\right|$ and $\overline{n_{i}}=n_{i-1}+n_{i}+n_{i+1}$. We define the deficiency $f_{i}$ of a distance layer $V_{i}$ by

$$
f_{i}=\delta+1-\overline{n_{i}} .
$$

Note that a vertex in $V_{i}$ has at least $f_{i}$ out-neighbours outside the set $V_{i-1} \cup V_{i} \cup V_{i+1}$.

Claim 1: for all $i \in\{1,2, \ldots, d-1\}$ we have $s \geqslant \max \left\{n_{i} f_{i}+n_{i+1} f_{i+1}, n_{i} f_{i}\right\}$.

Consider a vertex $w_{i} \in V_{i}$. Since $w_{i}$ has out-neighbours only in $V_{\leqslant i+1}$, we have

$$
\left|N^{+}\left(w_{i}\right) \cap V_{\leqslant i-1}\right| \geqslant d^{+}\left(w_{i}\right)-n_{i+1}-\left(n_{i}-1\right) \geqslant \delta-\overline{n_{i}}+n_{i-1}+1=f_{i}+n_{i-1} .
$$


Similarly for each $w_{i+1} \in V_{i+1}$,

$$
\left|N^{+}\left(w_{i+1}\right) \cap V_{\leqslant i-1}\right| \geqslant d^{+}\left(w_{i+1}\right)-\overline{n_{i+1}}+1 \geqslant \delta+1-\overline{n_{i+1}}=f_{i+1} .
$$

Combining the two inequalities yields

$$
q\left(V_{\geqslant i}, V_{\leqslant i-1}\right) \geqslant q\left(V_{i} \cup V_{i+1}, V_{\leqslant i-1}\right) \geqslant n_{i}\left(f_{i}+n_{i-1}\right)+n_{i+1} f_{i+i} .
$$

On the other hand we have

$$
q\left(V_{\leqslant i-1}, V_{\geqslant i}\right)=q\left(V_{i-1}, V_{i}\right) \leqslant n_{i-1} n_{i} .
$$

Since $D$ is $s$-eulerian, we obtain

$$
\begin{aligned}
s & \geqslant q\left(V_{\geqslant i}, V_{\leqslant i-1}\right)-q\left(V_{\leqslant i-1}, V_{\geqslant i}\right) \\
& \geqslant n_{i}\left(f_{i}+n_{i-1}\right)+n_{i+1} f_{i+i}-n_{i-1} n_{i} \\
& =n_{i} f_{i}+n_{i+1} f_{i+1}
\end{aligned}
$$

and so $s \geqslant n_{i} f_{i}+n_{i+1} f_{i+1}$. Similarly we prove $s \geqslant n_{i} f_{i}$.

Claim 2: If $n_{i}=1$ then

(a) if $0 \leqslant i \leqslant d-2$ then $f_{i+1} \geqslant f_{i+2}$ and $f_{i+2} \leqslant 0$,

(b) if $2 \leqslant i \leqslant d$ then $f_{i-1} \geqslant f_{i-2}$ and $f_{i-2} \leqslant 0$.

(a) From $\overline{n_{i+2}}=\overline{n_{i+1}}-n_{i}+n_{i+3} \geqslant \overline{n_{i+1}}$ we immediately get $f_{i+2} \leqslant f_{i+1}$. Now suppose to the contrary that $f_{i+2}>0$. Then $f_{i+1}>0$, and we obtain the contradiction

$s \geqslant n_{i+1} f_{i+1}+n_{i+2} f_{i+2} \geqslant\left(n_{i+1}-1\right)\left(f_{i+1}-1\right)+n_{i+1}+f_{i+1}-1+n_{i+2} \geqslant \overline{n_{i+1}}-2+f_{i+1}=\delta-1$.

(b) The proof uses the inequality $s \geqslant n_{i-1} f_{i-1}+n_{i-2} f_{i-2}$ and is analogous to part (a).

Clatm 3: Let $i \in\{1,2, \ldots, d-4\}$. If $n_{i}=1$ then either $f_{i}+f_{i+1}+f_{i+2}+f_{i+3} \leqslant s$ or we have, with $F_{i}:=f_{i}+f_{i+1}+f_{i+2}$ and $a:=n_{i-1}$,

$$
F_{i} \leqslant s, \quad n_{i+1} \leqslant \delta-a-F_{i}, \quad n_{i+2} \geqslant a+F_{i}
$$

Moreover, $F_{i}=s$ only if $f_{i-1} \leqslant 0$ and $n_{i+3}=1$.

First note that $f_{i+2} \leqslant 0$ by Claim 2 . We consider two cases.

Case 1: $f_{i+1} \leqslant 0$.

By Claim $1, s \geqslant n_{i} f_{i}=f_{i}$, so $F_{i}=f_{i}+f_{i+1}+f_{i+2} \leqslant s$ since $f_{i+1}, f_{i+2}$ are non-positive. If $F_{i}=s$ then $f_{i}=s$ and so, by $s \geqslant n_{i-1} f_{i-1}+n_{i} f_{i}$ we conclude that $f_{i-1} \leqslant 0$. Also $f_{i+1}=f_{i+2}=0$, and so $n_{i+3}=n_{i}=1$.

Let $n_{i-1}=a$. From the definition of the $\overline{n_{j}}$ and $f_{j}$ we immediately get $n_{i+1}=\delta-a-f_{i} \leqslant$ $\delta-a-F_{i}$ and $n_{i+2}=a+f_{i}-f_{i+1} \geqslant a+F_{i}$, as desired.

Case 2: $f_{i+1}>0$. 
If $n_{i+1}=1$, then $f_{i+3} \leqslant 0$ by Claim 2. Claim 1 now yields

$$
s \geqslant n_{i} f_{i}+n_{i+1} f_{i+1} \geqslant f_{i}+f_{i+1}+f_{i+2}+f_{i+3},
$$

as desired. So we assume that $n_{i+1} \geqslant 2$. Then $\overline{n_{i+3}}=\overline{n_{i+2}}-n_{i+1}+n_{i+4} \geqslant \overline{n_{i+2}}-n_{i+1}+1$. Hence we have $f_{i+3} \leqslant f_{i+2}+n_{i+1}-1 \leqslant n_{i+1}-1$. We also have $f_{i}+n_{i+1} f_{i+1} \leqslant s$ and thus

$$
f_{i}+f_{i+1}=f_{i}+n_{i+1} f_{i+1}-\left(n_{i+1}-1\right) f_{i+1} \leqslant s-n_{i+1}+1 .
$$

In total we obtain

$$
f_{i}+f_{i+1}+f_{i+2}+f_{i+3} \leqslant\left(s-n_{i+1}+1\right)+0+n_{i+1}-1=s,
$$

as desired.

Claim 4: Let $i \in\{1,2, \ldots, d-4\}$. If $n_{i}=2$ and $f_{i}>0$, then at least one of the following holds:

(i) $f_{i}+f_{i+1}+f_{i+2} \leqslant \frac{2}{3} s$,

(ii) $f_{i}+f_{i+1}+f_{i+2}+f_{i+3} \leqslant s$,

(iii) $f_{i}+f_{i+1}+f_{i+2}=\frac{1}{2} s+1, s \in\{2,4\}$ and $f_{i}=\frac{1}{2} s, f_{i+1}=0, f_{i+2}=1, n_{i}=2, n_{i+1} \geqslant 2$, $n_{i+2} \geqslant 2, n_{i+3}=1$

First note that $f_{i}>0$ implies that $s \geqslant n_{i} f_{i} \geqslant 2$ by Claim 1 and $n_{i+2} \geqslant 2$ by Claim 2 .

Case 1: $n_{i+1}=1$.

Then $f_{i+3} \leqslant 0$ and $\overline{n_{i+1}} \geqslant 5$ by Claim 2 . If $f_{i+2} \geqslant 2$, then we obtain the contradiction

$$
s \geqslant n_{i+1} f_{i+1}+n_{i+2} f_{i+2} \geqslant f_{i+1}+2 n_{i+2}=\delta+1-\overline{n_{i+1}}+2\left(\overline{n_{i+1}}-3\right) \geqslant \delta,
$$

so $f_{i+2} \leqslant 1$. Then $f_{i+2} \leqslant f_{i}$, and thus

$$
s \geqslant n_{i} f_{i}+n_{i+1} f_{i+1}=2 f_{i}+f_{i+1} \geqslant f_{i}+f_{i+1}+f_{i+2}+f_{i+3} .
$$

Case 2: $n_{i+1} \geqslant 2$.

Then $n_{i+2} \geqslant 2$ by Claim 2 and $f_{i}>0$.

If $n_{i+3} \geqslant 2$, then we obtain the claim by adding the inequalities $s \geqslant n_{i} f_{i}+n_{i+1} f_{i+1} \geqslant$ $2\left(f_{i}+f_{i+1}\right)$ and $s \geqslant n_{i+2} f_{i+2}+n_{i+3} f_{i+3} \geqslant 2\left(f_{i+2}+f_{i+3}\right)$, so we assume that $n_{i+3}=1$. By Claim 2 this implies $f_{i+1} \leqslant 0$. Note that $f_{i+2}=f_{i+1}+n_{i}-n_{i+3}=f_{i+1}+1$.

By Claim 1 we have $s \geqslant n_{i} f_{i}=2 f_{i}$, so $f_{i} \leqslant s / 2$. Now either $f_{i+1}=0$ or $f_{i+1} \leqslant-1$. If $f_{i+1} \leqslant-1$, then $f_{i+2} \leqslant 0$ and $f_{i}+f_{i+1}+f_{i+2} \leqslant \frac{1}{2} s-1<\frac{2}{3} s$, as desired. If $f_{i+1}=0$, then $f_{i+2}=1$, and thus $f_{i}+f_{i+1}+f_{i+2} \leqslant \frac{1}{2} s+1$. If the last inequality is strict, then $f_{i}+f_{i+1}+f_{i+2} \leqslant\left\lfloor\frac{s+1}{2}\right\rfloor \leqslant \frac{2}{3} s$. (Note that $s \geqslant 2$.) But if $f_{i}+f_{i+1}+f_{i+2}=\frac{1}{2} s+1$, then $s$ is even and $s<6$ since otherwise $\frac{s}{2}+1 \leqslant \frac{2}{3} s$.

Clatm 5: Let $i \in\{1,2, \ldots, d-4\}$. If $n_{i} \geqslant 3$ and $f_{i}>0$ then either $f_{i}+f_{i+1} \leqslant \frac{1}{3} s$ or $f_{i}+f_{i+1}+f_{i+2}+f_{i+3} \leqslant s$.

We can assume that $f_{i+1}>0$ since otherwise $s \geqslant n_{i} f_{i} \geqslant 3 f_{i}$ yields $f_{i}+f_{i+1} \leqslant s / 3$. We 
also have, by $f_{i}>0$ and Claim 2 , that $n_{i+2} \geqslant 2$.

Case 1: $n_{i+1}=1$.

Then $f_{i+3} \leqslant 0$ by Claim 2 . We can assume that $f_{i+2} \geqslant 0$ since otherwise $s \geqslant n_{i} f_{i}+$ $n_{i+1} f_{i+1} \geqslant f_{i}+f_{i+1}+f_{i+2}+f_{i+3}$ yields Claim 5. Now Claim 1 yields the inequalities

$$
s \geqslant 3 f_{i}+f_{i+1} \text { and } s \geqslant f_{i+1}+2 f_{i+2} \text {. }
$$

If $f_{i+2} \leqslant 2 f_{i}$, then the first inequality leads to $s \geqslant f_{i}+f_{i+1}+f_{i+2}$. Otherwise $2 f_{i}<f_{i+2}$ and thus $f_{i}<f_{i+2}$, and now the second inequality yields this bound.

Case 2: $n_{i+1} \geqslant 2$.

By our above assumption $f_{i+1}>0$, Claim 2 yields $n_{i+3} \geqslant 2$. Adding the two inequalities

$$
s \geqslant n_{i} f_{i}+n_{i+1} f_{i+1}>2\left(f_{i}+f_{i+1}\right), \text { and } s \geqslant n_{i+2} f_{i+2}+n_{i+3} f_{i+3} \geqslant 2\left(f_{i+2}+f_{i+3}\right)
$$

now yields Claim 5 .

The following claim follows immediately from Claims 3 to 5.

Claim 6: For each $i \in\{1,2, \ldots, d-4\}$ at least one of the following statements holds:

(i) $f_{i} \leqslant 0$,

(ii) $f_{i}+f_{i+1} \leqslant \frac{1}{3} s$

(iii) $f_{i}+f_{i+1}+f_{i+2} \leqslant \frac{2}{3} s$,

(iv) $f_{i}+f_{i+1}+f_{i+2}+f_{i+3} \leqslant s$

(v) $f_{i}+f_{i+1}+f_{i+2}=\frac{1}{2} s+1$ and $s \in\{2,4\}, n_{i}=2, n_{i+1} \geqslant 2, n_{i+2} \geqslant 2, n_{i+3}=1, f_{i}=\frac{1}{2} s$, $f_{i+1}=0, f_{i+2}=1$

(vi) $n_{i}=1$ and, with $F_{i}:=f_{i}+f_{i+1}+f_{i+2}$ and $a:=n_{i-1}$,

$$
F_{i} \leqslant s, \quad n_{i+1} \leqslant \delta-a-F_{i}, \quad n_{i+2} \geqslant a+F_{i}
$$

and $F_{i}=s$ only if $f_{i-1} \leqslant 0$ and $n_{i+3}=1$.

Let $I=\{a, a+1, \ldots, b-1\}$ be an interval. We will say that $I$ is of type (i) (type (ii), (iii), (iv), ( v), (vi)) if, with $i=a$, statement (i) (statement (ii), (iii), (iv), (v), (vi)) holds and $|I|=1(|I|=2,3,4,3,3)$. The following claim shows that each $j \in\{1,2, \ldots, d\}$ which is not too close to $d$, is a left end point of an interval $J$ for which $\sum_{i \in J} f_{i} \leqslant(|J|-1) \frac{s}{3}$.

Claim 7: If $s \leqslant \delta-2$ then $\sum_{i=1}^{d} f_{i} \leqslant \frac{d s}{3}+2 s$.

Repeated application of Claim 6 shows that we can partition the set $\{1,2, \ldots, d\}$ into intervals $I(1), I(2), \ldots, I(k)$ such that each interval, except possibly $I(k)$, is of one of the types in Claim 6 , and $I(k)$ has at most 3 elements.

It follows from Claim 6 and $\frac{1}{2} s+1 \leqslant s$ for $s \geqslant 2$, that for each interval $I(m)$, $m \in\{1,2, \ldots, k-1\}$,

$$
\sum_{i \in I(m)} f_{i} \leqslant \frac{s}{3}|I(m)| .
$$


Since $f_{i} \leqslant s$ for each $i \in I(k)$, we have

$$
\sum_{i \in I(k)} f_{i} \leqslant s|I(k)| \leqslant \frac{s}{3}|I(k)|+2 s .
$$

Hence

$$
\sum_{i=1}^{d} f_{i}=\sum_{m=1}^{k} \sum_{i \in I(m)} f_{i} \leqslant \sum_{m=1}^{k}|I(m)| \frac{s}{3}+2 s=\frac{d s}{3}+2 s
$$

as desired.

Claim 7 now implies part (a) of the theorem as follows. Clearly,

$$
\sum_{i=0}^{d} \overline{n_{i}}=3 n-n_{0}-n_{d} \leqslant 3 n
$$

On the other hand,

$$
\begin{aligned}
\sum_{i=0}^{d} \overline{n_{i}} & =\sum_{i=0}^{d}\left(\delta+1-f_{i}\right) \\
& \geqslant(d+1)(\delta+1)-\frac{d s}{3}-2 s
\end{aligned}
$$

Combining the inequalities and solving for $d$ now yields part (a) of the theorem.

Claim 8: Let $s$ divide $\delta-2$. If $j$ is fixed, $1 \leqslant j \leqslant d-3 \frac{\delta-2}{s}$, then there exists an integer $k$ with $k \leqslant j+3 \frac{\delta-2}{s}$ such that

$$
\sum_{i=j}^{k} f_{i} \leqslant(k-j) \frac{s}{3}
$$

There exist integers $j_{1}, j_{2}, \ldots, j_{r+1}$ with $j=j_{1}<j_{2}<\ldots<j_{r+1}$ and $j_{r}<j+3 \frac{\delta-2}{s} \leqslant j_{r+1}$ such that for each $m$ the interval $I(m):=\left\{j_{m}, j_{m}+1, j_{m}+2, \ldots, j_{m+1}-1\right\}$ is of one of the six types described in Claim 6 . It follows from Claim 6 and $\frac{1}{2} s+1 \leqslant s$ for $s \geqslant 2$, that

$$
\sum_{i \in I(m)} f_{i} \leqslant\left\{\begin{array}{cl}
\frac{1}{3} s(|I(m)|-1) & \text { if } I(m) \text { is of type (i), (ii), (iii), or (iv), } \\
\frac{1}{3} s|I(m)| & \text { if } I(m) \text { is of type (v) or (vi). }
\end{array}\right.
$$

Case 1: $I(1)$ is of type $(\mathrm{v})$.

First consider the case $s=\delta-2$. If $s=2$ then $\delta=4$, but $n_{i}+n_{i+1}+n_{i+2} \geqslant 2+2+2 \geqslant 6>$ $\delta+1$ by Claim 4 , contradicting $f_{i+1}=0$. If $s=4$ then $\delta=6$ and, by Claim $4, f_{i}=\frac{1}{2} s=2$. Hence, $n_{i+1}=2$ since otherwise, if $n_{i+1}>2$ we have the contradiction $\overline{n_{i}} \geqslant 6=\delta+1$. But then $f_{i+2}=1$ implies $n_{i+2}=3$. By Claim 1 we get $4=s \geqslant n_{i+2} f_{i+2}+n_{i+3} f_{i+3}=3+f_{i+3}$, so $f_{i+3}=1$. Hence we have $f_{i}+f_{i+1}+f_{i+2}+f_{i+3} \leqslant 4=s$, and Claim 8 holds with $k=j+3$. 
Now consider the case $s<\delta-2$. Consider $I(2)$. By Claim 4 we have $n_{i+3}=1$, so $I(2)$ is not of type (v). If $I(2)$ is of type (i), (ii), (iii), or (iv), then (1) implies

$$
\sum_{i \in I(1) \cup I(2)} \leqslant(|I(1) \cup I(2)|-1) \frac{s}{3},
$$

and Claim 8 follows with $k=j_{3}-1$. If $I(2)$ is of type (vi), then it follows from Claim 3 and $f_{j+2}=1$ that $f_{j+3}+f_{j+4}+f_{j+5} \leqslant s-1$, and so

$$
\sum_{i=j}^{j+5} f_{i} \leqslant \frac{1}{2} s+1+s-1 \leqslant 5 \frac{s}{3}
$$

and Claim 8 holds with $k=j+5$.

Case 2: $I(1), I(2), \ldots, I(m-1)$ are of type (vi) and $I(m)$ is of type (v) for some $m$ with $2 \leqslant m \leqslant r$.

Consider $I(m-1)$. Since $n_{j_{m}}=2$, it follows by Claim 3 that $\sum_{i \in I(m-1)} \leqslant s-1$. Hence,

$$
\sum_{i=j}^{j_{m+1}-1} f_{i} \leqslant s(m-1)+s-1+\frac{1}{2} s+1 \leqslant\left(j_{m+1}-1-j\right) \frac{s}{3},
$$

and Claim 8 follows with $k=j_{m+1}-1$.

Case 3: $I(m)$ is of type (i), (ii), (iii) or (iv) for some $m \leqslant r$.

Let $I(m)$ be the first interval of type of type (i), (ii), (iii), or (iv). By (1) we have

$$
\sum_{i=j}^{j_{m+1}-1} f_{i}=\sum_{k=0}^{m-1} \sum_{i \in I(k)} f_{i}+\sum_{i \in I(m)} f_{i} \leqslant \sum_{k=0}^{m-1}|I(k)| \frac{s}{3}+(|I(m)|-1) \frac{s}{3}=\left(j_{m+1}-1-j\right) \frac{s}{3} .
$$

To prove Claim 8 for this case it remains to show that $j_{m+1}-1 \leqslant j+3 \frac{\delta-2}{s}$. Since intervals $I(1), I(2), \ldots, I(m)$ have 3 elements each, we have $j_{m}=j+3(m-1)$, and thus $j_{m} \equiv j+3 \frac{\delta-2}{s} \quad(\bmod 3)$. By $j_{m}<j+3 \frac{\delta-2}{s}$ we obtain $j_{m} \leqslant j+3 \frac{\delta-2}{s}-3$. Since $j_{m+1}$ has at most four elements, we have $j_{m+1} \leqslant j_{m}+4 \leqslant j+3 \frac{\delta-2}{s}+1$, as desired.

Case 4: $I(m)$ is of type (vi) for $m=0,1, \ldots, r$.

Since each interval contains exactly 3 numbers, we have $r=\frac{\delta-2}{s}$ and so $j_{r+1}=j+3 \frac{\delta-2}{s}$. As in Claim 3 we let $F_{i}=f_{i}+f_{i+1}+f_{i+2}$. Then we have

$$
\sum_{i=j}^{j+3 \frac{\delta-2}{s}-1} f_{i}=\sum_{i=0}^{\frac{\delta-2}{3}} F_{j+3 i}
$$

Clearly $n_{j-1} \geqslant 1$. Applying statement (vi) iteratively, we obtain $n_{j+2} \geqslant n_{j-1}+F_{j} \geqslant 1+F_{j}$, $n_{j+5} \geqslant n_{j+2}+F_{j+3} \geqslant 1+F_{j}+F_{j+3}$, and so on. Finally,

$$
n_{j+3 \frac{\delta-2}{s}-1} \geqslant 1+\sum_{i=0}^{\frac{\delta-2}{s}-1} F_{j+3 i}=1+\sum_{i=j}^{j+3 \frac{\delta-2}{s}-1} f_{i} .
$$


Hence, since each distance layer has at least one vertex,

$$
\overline{n_{j+3 \frac{\delta-2}{s}-1}} \geqslant 3+\sum_{i=j}^{j+3 \frac{\delta-2}{s}-1} f_{i},
$$

and so

$$
f_{j+3 \frac{\delta-2}{s}} \leqslant \delta-2-\sum_{i=j}^{j+3 \frac{\delta-2}{s}-1} f_{i}
$$

or equivalently,

$$
\sum_{i=j}^{j+3 \frac{\delta-2}{s}} f_{i} \leqslant \delta-2=3 \frac{\delta-2}{s} \frac{s}{3},
$$

and Claim 8 follows with $k=j+3 \frac{\delta-2}{s}$.

Claim 9: If $s$ divides $\delta-2$ then $\sum_{i=0}^{d} f_{i} \leqslant d \frac{3(\delta-2)}{3(\delta-2)+s}+\frac{s(\delta-2)}{3(\delta-2)+s}+2 s$.

We partition most of the interval $\{1,2, \ldots, d\}$ into subintervals to which we apply Claim 8 . Repeated application of Claim 8 now shows that there exist integers $k(0)<k(1) \ldots<k(t)$ such that $k(0)=1, k(t)>d-3 \frac{\delta-2}{s}, k(j+1)-k(j) \leqslant 3 \frac{\delta-2}{s}+1$ for $j=0,1, \ldots, t-1$, and

$$
\sum_{i=k(j)}^{k(j+1)-1} f_{i} \leqslant(k(j+1)-k(j)-1) \frac{s}{3} .
$$

Summation over all $j \in\{0,1, \ldots, t-1\}$ yields

$$
\sum_{i=1}^{k(t)-1} f_{i} \leqslant(k(t)-1) \frac{s}{3}-t \frac{s}{3} .
$$

Since $k(j+1)-k(j) \leqslant 3 \frac{\delta-2}{s}+1$, we bound $t$ from below by

$$
\begin{aligned}
t & \geqslant(k(t)-1)\left[3 \frac{\delta-2}{s}+1\right]^{-1} \\
& \geqslant\left(d-3 \frac{\delta-2}{s}\right) \frac{s}{3(\delta-2)+s} \\
& =d \frac{s}{3(\delta-2)+s}-\frac{3(\delta-2)}{3(\delta-2)+s} .
\end{aligned}
$$

We now bound the sum of the remaining $f_{i}$ at the left and right end of the set $\{0,1, \ldots, d\}$. We have $f_{0} \leqslant 0$ since $N^{+}(v) \subseteq V_{0} \cup V_{1}$ and so $\overline{n_{0}}=n_{0}+n_{1} \geqslant \delta+1$. We partition the set $\{k(t), k(t)+1, \ldots, d\}$ into intervals $F_{1}, F_{2}, \ldots, F_{r}$ such that each $F_{j}, j \leqslant r-1$, is of one of 
the types in Claim 6 , and $F_{r}$ has at most 3 elements. As in (1), we have $\sum_{i \in F_{j}} f_{i} \leqslant\left|F_{j}\right| \frac{s}{3}$ for $j \leqslant r-1$. Also $\sum_{i \in F_{r}} f_{i} \leqslant\left|F_{r}\right| s$. Hence we obtain

$$
\sum_{i=k(t)}^{d} f_{i} \leqslant(d+1-k(t)) \frac{s}{3}+2 s=d \frac{3(\delta-2)}{3(\delta-2)+s}+\frac{s(\delta-2)}{3(\delta-2)+s}+2 s
$$

which is Claim 9.

Part (b) of the theorem now follows exactly as part (a) does after Claim 7.

We now show that the coefficient in the above bound is best possible. Let $a>0$ be an integer. By $K_{a}$ we mean the complete directed graph on $a$ vertices and $a(a-1)$ arcs. For vertex disjoint digraphs $D_{1}, D_{2}, \ldots, D_{k}$ we define the sequential sum $D_{1}+D_{2}+\ldots+D_{k}$ to be the digraph obtained from the union of the $D_{i}$ by joining each vertex in $D_{i}$ to and from each vertex in $D_{i+1}$ for $i=1,2, \ldots, k-1$.

Let $\delta, s$ be given, and let $s$ divide $\delta-2$. From the following digraph $H^{\prime}$, which is a sequential sum of $3 \frac{\delta-2}{s}+2$ complete digraphs, we will obtain the main building block.

$$
\begin{aligned}
H^{\prime}= & K_{1}+K_{\delta-1-s}+K_{1+s}+K_{1}+K_{\delta-1-2 s}+K_{1+2 s}+K_{1}+K_{\delta-1-3 s}+K_{1+3 s} \\
& +\ldots+K_{1}+K_{1+s}+K_{\delta-1-s}+K_{1}+K_{1}+K_{\delta-1}+K_{1}+K_{1} .
\end{aligned}
$$

Let the digraph $H$ be obtained from $H^{\prime}$ as follows. Denote the complete digraphs in the above sequential sum by $D_{0}, D_{1}, \ldots, D_{3 \frac{\delta-2}{s}+1}$. For $j=0,1,2, \ldots, \frac{\delta-2}{s}-2$ let $v^{3 j}$ be the vertex in $D_{3 j}$, and choose distinct vertices $u_{1}^{3 j+1}, u_{2}^{3 j+1}, \ldots, u_{s}^{3 j+1} \in D_{3 j+1}$ and $w_{1}^{3 j+2}, w_{2}^{3 j+2}, \ldots, w_{s}^{3 j+2} \in D_{3 j+2}$. Also let $v^{3 \frac{\delta-2}{s}-2}$ and $v^{3 \frac{\delta-2}{s}+1}$ be the vertex in $D_{3 \frac{\delta-2}{s}-2}$ and $D_{3 \frac{\delta-2}{s}+1}$, respectively. For $i=1,2, \ldots, s$ remove the arc $w_{i}^{3 j+2} u_{i}^{3 j+1}$ and add the arcs $v^{3 j+3} u_{i}^{3 j+1}$ and $w_{i}^{3 j+2} v^{3 j}$. Furthermore, join vertex $v^{3 \frac{\delta-2}{s}+1}$ to $s$ distinct vertices in $D_{3 \frac{\delta-2}{s}-1}$ and join these to vertex $v^{3 \frac{\delta-2}{s}-3}$. It is easy to see that for each vertex in $H$ its in-degree equals its out-degree, and both are at least $\delta$, except for $v^{0}$ and $v^{3 \frac{\delta-2}{s}+1}$. The diagram shows the digraph $H$ for $\delta=8$ and $s=2$. An undirected edge between two vertices $a$ and $b$ stands for the two arcs $a b$ and $b a$. Note that each $D_{i}$ is complete, but arcs between vertices of the same $D_{i}$ are not shown.

Now let $H_{1}, H_{2}, \ldots, H_{k}$ be disjoint copies of $H$. Let $a_{i}$ and $b_{i}$ be vertex $v^{0}$ and $v^{3 \frac{\delta-2}{s}+1}$, respectively, of $H$. We define the digraph $D$ as the graph obtained from the union of $H_{1}, H_{2}, \ldots, H_{k}$ and two complete digraphs $K_{\delta+1}^{\prime}$ and $K_{\delta+1}^{\prime \prime}$, by identifying $b_{i}$ with $a_{i+1}$ for $i=1,2, \ldots, k-1$, and joining vertex $a_{1}$ to and from all vertices in $K_{\delta+1}^{\prime}$, and $b_{k}$ to and from all vertices in $K_{\delta+1}^{\prime \prime}$.

It is easy to verify that the obtained digraph $D$ has minimum degree $\delta$ and that it is $s$-eulerian, and that, for large $k$ and constant $s$ and $\delta$,

$$
\operatorname{diam}(D)=3\left(\delta+1-\frac{(\delta-2) s}{3(\delta-2)+s}\right)^{-1} n+O(1)
$$


Corollary 1 Let $D$ be a strong semi-eulerian digraph of order $n$ and minimum degree $\delta$. Then

$$
\operatorname{diam}(D) \leqslant\left(\delta+1-\frac{\delta-2}{3 \delta-5}\right)^{-1} n+O(1)
$$

Apart from the additive constant this bound is best possible.

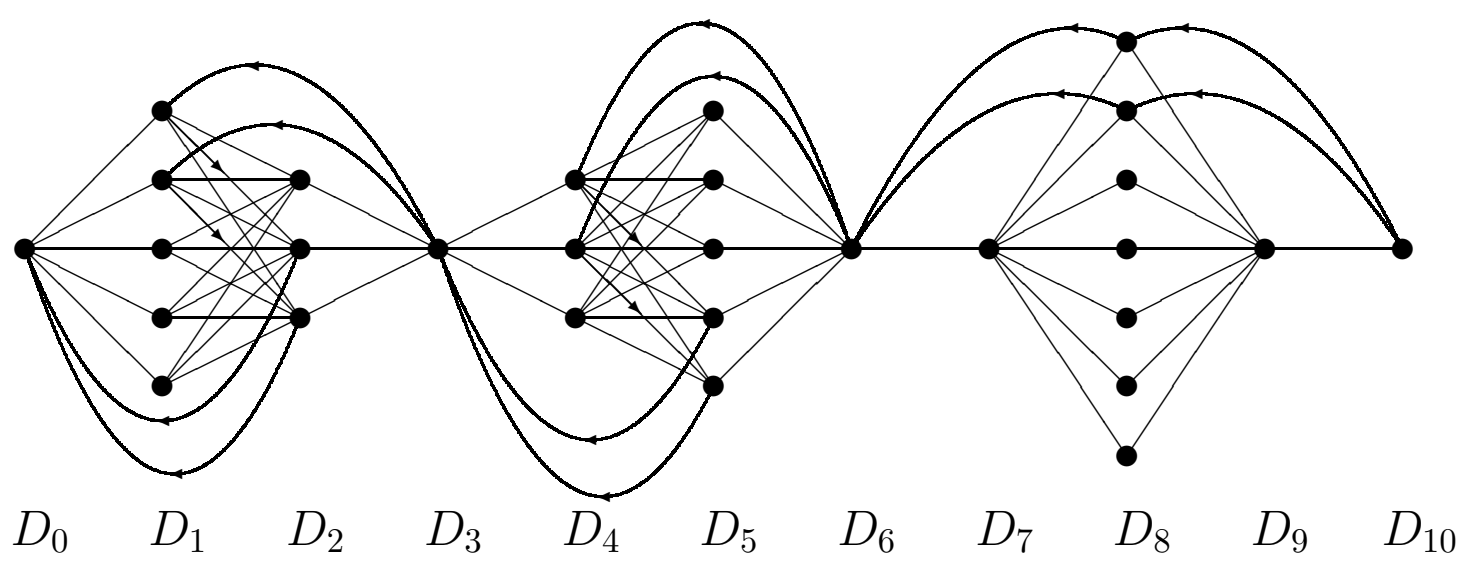

Remark (i) Theorem 2(b) and the above example show that $c_{\delta, s}=3\left(\delta+1-\frac{(\delta-2) s}{3(\delta-2)+s}\right)^{-1}$ if $s$ divides $\delta-2$. Theorem 2 (a) only yields the upper bound $c_{\delta, s} \leqslant 3\left(\delta+1-\frac{s}{3}\right)^{-1}$. We do not believe that equality holds, but we were unable to determine the exact value of $c_{\delta, s}$. (ii) The determination of the values for $c_{\delta, s}$ for $s>\delta-2$ will be considered elsewhere. We just state here, without proof, that $c_{\delta, s}<1$ if and only if $s \leqslant\left(\begin{array}{l}\delta \\ 2\end{array}\right)-2$.

\section{References}

[1] P. Dankelmann, The diameter of directed graphs. Journal of Combinatorial Theory B 94 (2005), 183-186.

[2] A.V. Knyazev, Diameters of Pseudosymmetric Graphs (Russian), Mat. Zametki 41 no. 6 (1987) 829-843. (English translation: Math. Notes 41 no. 5-6 (1987) 473-482.)

[3] J. Soares, Maximum diameter of regular digraphs. J. Graph Theory 16 no. 5 (1992) 437-450. 